\title{
ON THE STARTING PROCESS \\ OF STRONGLY NONLINEAR VORTEX/RAYLEIGH-WAVE INTERACTIONS
}

\author{
P. G. BROWN, S. N. BROWN, F. T. SMITH AND S. N. TIMOSHIN
}

\begin{abstract}
An oncoming two-dimensional laminar boundary layer that develops an unstable inflection point and becomes three-dimensional is described by the Hall-Smith (1991) vortex/wave interaction equations. These equations are now examined in the neighbourhood of the position where the critical surface starts to form. A consistent structure is established in which an inviscid core flow is matched to a viscous buffer-layer solution where the appropriate jump condition on the transverse shear stress is satisfied. The final result is a bifurcation equation for the (constant) amplitude of the wave pressure. A representative classical velocity profile is considered to illustrate solutions of this equation for a range of values of the wave-numbers.
\end{abstract}

\$1. Introduction. Vortex/wave interaction, for the flow in a boundary layer, pipe or channel, for example, concerns the nonlinear interplay between the mean vortex part, comprising longitudinal or streamwise vortices of relatively long scale, and the wave part which generally has shorter length scales. The theory of vortex/wave interactions is the third strongly nonlinear one to be developed for describing rationally some central aspects of transition to turbulence, in flows over flat surfaces, following the earlier theories of pressuredisplacement interaction (for instance triple-deck) and of Euler-scale motion. See references below. In strongly nonlinear vortex/wave interactions, the vortex part constitutes the entire mean flow, whereas in weakly nonlinear interactions the vortex part is a small-amplitude perturbation of the mean flow. Our concern here is with the starting process of the strongly nonlinear case. This is currently attracting much interest because of the remarkably low amplitudes of the three-dimensional wave disturbances that can provoke a relative effect of order unity in the total (three-dimensional) mean flow, as described later.

Two main types of nonlinear wave contribution have been considered previously, namely Tollmien-Schlichting and Rayleigh waves. Of these, the former has received more attention so far, through the studies of weakly nonlinear interactions in Hall and Smith $(1988,1989,1990)$, Smith and Walton (1989), Blackaby (1991), Smith and Blennerhassett (1992) and of strongly nonlinear interactions in Hall and Smith (1991), Smith and Walton (1989), Walton (1991), Walton and Smith (1992), covering a range of incompressible and compressible boundary layers and incompressible channel flows. We note in passing that the weak interactions, generally involving shorter vortices, are 
found to develop into the strong ones occasionally but far from always. The nonlinear interaction between vortices and Rayleigh waves, in contrast, has received scant attention to date: see Hall and Smith (1991), Davis (1922). Yet the vortex/Rayleigh-wave case is in a sense the more interesting and dangerous, in practice, in that it is initiated by much tinier disturbances and these are more common and would be difficult to eradicate as regards control of transition for example. This gap in understanding in fact prompts the current theoretical studies of nonlinear vortex/Rayleigh-wave interaction, in bound ary-layer motions, first for longer-scale stronger interactions (below) and then for the shorter-scale weaker interactions (Smith et al., 1992) that are implied by the present work. The work, like others above, is believed to be particularly relevant to fully fledged transition in boundary layers; the first firm comparisons, made recently by Stewart and Smith (1992), show encouraging agreement in qualitative and quantitative terms between their interaction analysis and the experiments of Klebanoff and Tidstrom (1959).

Our particular interest is in the way that a strongly nonlinear vortex/ Rayleigh-wave interaction starts, in the spatial sense ( $c f$. the terminal forms investigated by Walton and Smith, 1992). This is partly because of the intrinsic interest, and because more insight is needed, but more especially to discover whether (or not) the nonlinear interaction solution can start, i.e., formally exist, and where and under what conditions, for the incident mean flow and the input wave(s). Such analysis with its emphasis on the flow structure is felt likely to yield more fresh insight anyway than would a direct computation. Indeed, the present analysis is found to point quite directly to the new form of vortex/wave interaction, on shorter length scales, addressed in the subsequent work of Smith et al. (1992). There it is shown that the travelling-wave solution considered here is just one of the possible situations to which the flow on the shorter length scale can evolve.

The scales and governing equations of the vortex/Rayleigh-wave interaction are presented in Section 2 below (with an appendix describing the critical-layer response), for an inçompressible boundary layer. A wide range of scales is dealt with, covering nondimensional streamwise lengths $x$ (based on airfoil chord, say) from the order of $R^{-3 / 4}$ to order unity for the vortex part, where the global Reynolds number $R$ is large, and including interactive and noninteractive boundary layers. Sections 3-8 then address the starting behaviour of the nonlinear three-dimensional interaction, given a two-dimensional oncoming mean flow with an inflectional streamwise velocity profile at the initial station $x=0$ accompanied by linearly neutral Rayleigh waves there. The mean or vortex flow and the wave amplitude just beyond, for small positive $x$, have to adjust by means of interaction to keep the nonlinear waves neutral. The adjustment induces a rather rich and interesting flow structure locally, as described in Sections 3 and 4 for the vortex and wave respectively in the majority of the boundary layer, and in Sections 5 and 6 concerning in turn the vortex and the wave in a thinner viscous buffer zone provoked near the incident critical level. The analysis indicates how the inflection-point condition is gradually eroded, as $x$ increases and interaction settles in, with the critical layer gradually acquiring a three-dimensional corrugated appearance. The analysis also leads to the governing equation for the wave amplitude, given 
in Section 7, while in Section 8 we consider the specific values of amplitude produced by a representative input velocity profile, which among other things verifies explicitly that starting solutions are possible for the nonlinear interaction. Further brief comments are presented in Section 9.

§2. The Hall-Smith vortex-wave interaction equations. These equations were obtained by Hall and Smith (1991) and apply to a variety of boundarylayer flows. They are derived from the three-dimensional unsteady NavierStokes equations as follows and are quoted here for the incompressible case. The dimensional starred variables, lengths $\left(x^{*}, y^{*}, z^{*}\right)$, Cartesian velocity components $\left(u^{*}, v^{*}, w^{*}\right)$, pressure/density ratio $p^{*} / \rho^{*}$ and time $t^{*}$ are first made non-dimensional with a representative length $L^{*}$, representative speed $U^{*}, U^{* 2}$ and $L^{*} / U^{*}$ respectively. Thus

$$
\begin{gathered}
(x, y, z)=\left(x^{*}, y^{*}, z^{*}\right) / L^{*}, \quad(u, v, w)=\left(u^{*}, v^{*}, w^{*}\right) / U^{*}, \\
p=p^{*} / \rho^{*} U^{* 2}, \quad t=t^{*} U^{*} / L^{*} .
\end{gathered}
$$

The Reynolds number $R$, which is taken to be large, is defined as

$$
R=U^{*} L^{*} / \nu^{*}
$$

where $\nu^{*}$ is the kinematic viscosity of the fluid.

The flow that, from upstream, approaches the region in which the vortexwave interaction is to take place is taken to be a steady two-dimensional $z$-independent boundary-layer flow in the $x$-direction over a wall at $y=0$. This can be generalized to $y=g(x)$ to accommodate flow over a hump but we shall adhere to the simpler situation here. The order of the thickness of the boundary layer in terms of the inverse of the Reynolds number need not be prescribed nor need the streamwise scale on which this boundary layer varies, -although some restrictions must be satisfied as explained below. Thus we write

$$
y=R^{-\delta} \bar{y}, \quad x=R^{-b} \bar{x},
$$

where we shall require, for consistency of the approximations to be made, that $0<\delta$ and $\delta>b$. The second of these requirements implies that variations across the boundary layer are more rapid than those along it, as is satisfied, for example, by the classical Prandtl boundary layer for which $\delta=\frac{1}{2}$ and $b=0$. Other examples are given below.

The vortex-wave interaction equations are obtained by superimposing a wave-like component upon this boundary-layer flow but in such a manner that the basic flow itself is altered and becomes three-dimensional and unknown. It emerges that, for this to happen, the original two-dimensional flow must develop an inflection point and become unstable to inviscid, Rayleigh-type, three-dimensional perturbations. The wave-like component of the flow is characterized by a rapid oscillation on an $x$-scale comparable with the boundary-layer thickness and a time-scale such that disturbances travel with a speed that is of the same order as that of the streamwise flow in the boundary layer. Thus we define

$$
E=\exp \left[i\left(\alpha_{0} X-\Omega T\right)\right]
$$


where $\Omega$ is a prescribed real frequency, $T=R^{3 \delta-b-1} t$, and

$$
\alpha_{0} X=R^{\delta-b} \int \alpha(\bar{x}) d \bar{x} .
$$

Here $\alpha(\bar{x})$ is also real and to be determined. With a scaling in the third dimension so that the $z$-variation is of the same order as that in the direction normal to the wall we have

$$
z=R^{-\delta} \bar{z}
$$

and the Hall-Smith velocity components and pressure are written as

$$
\begin{gathered}
u=R^{-b-1+2 \delta}\left\{\bar{u}(\bar{x}, \bar{y}, \bar{z})+R^{-7(\delta-b) / 6}(\tilde{u}(\bar{x}, \bar{y}, \bar{z}) E+\text { c.c. })\right\} \\
\left(\begin{array}{c}
v \\
w
\end{array}\right)=R^{-1+\delta}\left\{\left(\begin{array}{c}
\bar{v}(\bar{x}, \bar{y}, \bar{z}) \\
\bar{w}(\bar{x}, \bar{y}, \bar{z})
\end{array}\right)+R^{-(\delta-b) / 6}\left(\begin{array}{c}
\tilde{v}(\bar{x}, \bar{y}, \bar{z}) \\
\tilde{w}(\bar{x}, \bar{y}, \bar{z})
\end{array}\right) E+\text { c.c. }\right\}, \\
p=R^{-2 b-2+4 \delta}\left\{\bar{p}(\bar{x})+R^{-2(\delta-b)} \bar{q}(\bar{x}, \bar{y}, \bar{z})+R^{-7(\delta-b) / 6}(\tilde{p}(\bar{x}, \bar{y}, \bar{z}) E+\text { c.c. })\right\} .
\end{gathered}
$$

In (2.7) the terms $\bar{u}, \tilde{u}$ etc. are the leading order contributions to the vortex ( $E$-independent) and wave ( $E$-dependent) terms respectively. The complex conjugate is denoted by c.c. As we shall see below, various familiar situations in classical and interactive boundary layers are recovered by assigning particular values to $\delta$ and $b$. The power of $R$, namely $R^{-(\delta-b) / 6}$, that is evident as a multiplier of the wave terms, is determined by the solution in the critical layer.

The Hall-Smith vortex-wave interaction equations are given in their 1991 paper and are as follows:

$$
\begin{gathered}
\frac{\partial \bar{u}}{\partial \bar{x}}+\frac{\partial \bar{v}}{\partial \bar{y}}+\frac{\partial \bar{w}}{\partial \bar{z}}=0, \\
\bar{u} \frac{\partial \bar{u}}{\partial \bar{x}}+\bar{v} \frac{\partial \bar{u}}{\partial \bar{y}}+\bar{w} \frac{\partial \bar{u}}{\partial \bar{z}}=-\bar{p}^{\prime}(\bar{x})+\frac{\partial^{2} \bar{u}}{\partial \bar{y}^{2}}+\frac{\partial^{2} \bar{u}}{\partial \bar{z}^{2}}, \\
\bar{u} \frac{\partial \bar{v}}{\partial \bar{x}}+\bar{v} \frac{\partial \bar{v}}{\partial \bar{y}}+\bar{w} \frac{\partial \bar{v}}{\partial \bar{z}}=-\frac{\partial \bar{q}}{\partial \bar{y}}+\frac{\partial^{2} \bar{v}}{\partial \bar{y}^{2}}+\frac{\partial^{2} \bar{v}}{\partial \bar{z}^{2}}, \\
\bar{u} \frac{\partial \bar{w}}{\partial \bar{x}}+\bar{v} \frac{\partial \bar{w}}{\partial \bar{y}}+\bar{w} \frac{\partial \bar{w}}{\partial \bar{z}}=-\frac{\partial \bar{q}}{\partial \bar{z}}+\frac{\partial^{2} \bar{w}}{\partial \bar{y}^{2}}+\frac{\partial^{2} \bar{w}}{\partial \bar{z}^{2}},
\end{gathered}
$$

for the vortex, and Rayleigh's equation

$$
(\bar{u}-\bar{c}(\bar{x}))\left(\frac{\partial^{2} \tilde{p}}{\partial \bar{y}^{2}}+\frac{\partial^{2} \tilde{p}}{\partial \bar{z}^{2}}-\alpha^{2} \tilde{p}\right)-2\left(\frac{\partial \tilde{p}}{\partial \bar{y}} \frac{\partial \bar{u}}{\partial \bar{y}}+\frac{\partial \tilde{p}}{\partial \bar{z}} \frac{\partial \bar{u}}{\partial \bar{z}}\right)=0,
$$

for the wave. Here $\bar{c}(\bar{x})=\Omega / \alpha(\bar{x})$ and is real. The boundary conditions associated with (2.8), (2.9) are that, as $\bar{y} \rightarrow 0, \bar{u}, \bar{v}, \bar{w}$ and $\partial \tilde{p} / \partial \bar{y}$ all tend to zero while the conditions as $\bar{y} \rightarrow \infty$ depend on the problem under consideration which determines the type of boundary layer that is developing the unstable inflection point. For example, for a classical boundary layer we have $b=0$, 
$\delta=\frac{1}{2}$ in $(2.7), \bar{p}^{\prime}(\bar{x})$ in $(2.8 \mathrm{~b})$ is prescribed and

$$
\bar{u} \frac{\partial \bar{u}}{\partial \bar{x}} \rightarrow-\bar{p}^{\prime}(\bar{x}), \quad \bar{w} \rightarrow 0 \quad \text { as } \quad \bar{y} \rightarrow \infty .
$$

For an interactive boundary layer of standard triple-deck type we have $b=\frac{3}{8}$, $\delta=\frac{5}{8}$ in $(2.7)$ and

$$
\bar{u} \sim \bar{y}+\bar{A}(\bar{x}), \quad \bar{w} \rightarrow 0 \quad \text { as } \quad \bar{y} \rightarrow \infty,
$$

where

$$
\bar{p}(\bar{x})=\frac{1}{\pi} \int_{-\infty}^{\infty} \frac{\bar{A}^{\prime}(\bar{\xi})}{\bar{x}-\bar{\xi}} d \bar{\xi}
$$

On the other hand if we take $b=-1+5 \delta / 2$ with $b<0$ we recover the hydraulic jump situation of Gajjar and Smith (1983) for which (2.12) is replaced by

$$
\bar{p}(\bar{x})=-\bar{A}(\bar{x}) \text {. }
$$

Similar replacements hold for other interactive flows (e.g., if $\delta=\frac{1}{2}+\frac{1}{3} b$ with $\frac{3}{8}<b<\frac{3}{4}$, then $\bar{A} \equiv 0$ instead of (2.12)). In all cases, however, $\tilde{p} \rightarrow 0$ as $\bar{y} \rightarrow \infty$.

The current study is concentrated on the development of the flow in the neighbourhood of the critical level, namely the (unknown) curve $\bar{y}=f(\bar{x}, \bar{z})$ at which $\bar{u}(\bar{x}, \bar{y}, \bar{z})=\bar{c}(\bar{x})$, so the conditions as $\bar{y} \rightarrow 0$ and $\bar{y} \rightarrow \infty$ are not of great import. However the further linking between $(2.8),(2.9)$ is, as shown by Hall and Smith, crucial and its form is independent of which of the above-mentioned problems we choose to examine. The further requirements are that, at the critical level $\bar{y}=f(\bar{x}, \bar{z}), \bar{u}, \bar{v}, \partial \bar{u} / \partial \bar{y}$ are continuous as is $\partial^{2} \bar{u} / \partial \bar{y}^{2}$. On the other hand, the shear stresses in the $\bar{y}, \bar{z}$ directions are discontinuous at $\bar{y}=f(\bar{x}, \bar{z})$ with

$$
\left[\frac{\partial \bar{w}}{\partial \bar{y}}\right]^{+}=J(\bar{x}, \bar{z})=\frac{1}{f_{\bar{z}}}\left[\frac{\partial \bar{v}}{\partial \bar{y}}\right]^{+}
$$

where

$$
J(\bar{x}, \bar{z})=\frac{\left(\frac{2}{3}\right)^{2 / 3}\left(-\frac{2}{3}\right) ! 2 \pi}{\Delta^{5} a^{5 / 3}}\left\{\left(-\frac{7 \Delta_{\bar{z}}}{2 \Delta}-\frac{5 a_{\bar{z}}}{3 a}\right)\left|\tilde{p}_{\bar{z}}\right|^{2}+\frac{\partial}{\partial \bar{z}}\left(\left|\tilde{p}_{\bar{z}}\right|^{2}\right)\right\} .
$$

In (2.14), $\Delta=1+f_{\bar{z}}^{2}, a=\bar{\lambda} \alpha / \Delta$ where $\bar{\lambda}(\bar{x}, \bar{z})$ is the unknown streamwise shear stress at the critical level, i.e., $\bar{\lambda}(\bar{x}, \bar{z})=\partial \bar{u} / \partial \bar{y}$ at $\bar{y}=f(\bar{x}, \bar{z})$. The square brackets in $(2.14 \mathrm{a})$ denote that it is the jump in the induced cross-flow shear stress that is related to the cross-flow derivatives of the wave pressure. In the Appendix we discuss the critical layer, i.e., the neighbourhood of the critical level where $\bar{y}-f(\bar{x}, \bar{z})=O\left(R^{-(\delta-b) / 3}\right)$, with slightly more detail than did Hall and Smith. The pressure function $\bar{q}$ in $(2.8 \mathrm{c}, \mathrm{d})$ is also discontinuous at the critical surface as is shown in the Appendix. However, the prescribed jump in $\bar{q}$ is relatively small in the starting process and hence does not affect the solution below.

In the following sections we examine the development of the flow in the neighbourhood of the point, $\bar{x}=0$ say, at which the vortex-wave interaction is assumed to start. For $\bar{x}<0$ the solutions of (2.8) are independent of $\bar{z}$, and the only solution of (2.9) subject to the required boundary conditions is $\tilde{p}=0$ 
even if the profile $\bar{u}$ has an inflection point. At $\bar{x}=0$, however, a point of neutral stability is attained, an eigensolution to (2.9) exists and the critical level is initiated. The mean flow then develops so that neutrality persists, and we now describe the flow in the neighbourhood of the leading edge of this critical level with $0<\bar{x} \ll 1$.

\$3. The core solution for the vortex. It emerges that, when $0<\bar{x} \ll 1$, the solution of equations (2.8), (2.9) must be considered in two main regions, namely $\bar{y}-f(\bar{x}, \bar{z})=O(1)$ and $\bar{y}-f(\bar{x}, \bar{z})=O\left(\bar{x}^{1 / 2}\right)$, in addition to a passive viscous layer in the neighbourhood of the wall. The regions above and below the critical level which have $\bar{y}-f(\bar{x}, \bar{z})=O(1)$ we term the core regions, and those in which $\bar{y}-f(\bar{x}, \bar{z})=O\left(\bar{x}^{1 / 2}\right)$ the buffer regions. See Fig. 1. The buffer, or diffusion, regions are separated by the critical layer, to which equations (2.8), (2.9) do not apply, but which furnishes the boundary conditions (2.14). At $\bar{x}=0$ the flow is $z$-independent with a streamwise velocity profile $U_{0}(\bar{y})$ that has an inflection point at $\bar{a}_{0}$ so that, near $\bar{y}=\bar{a}_{0}$,

$$
U_{0}(\bar{y})=c_{0}+b_{1}\left(\bar{y}-\bar{a}_{0}\right)+\sum_{n=3}^{\infty} \frac{b_{n}\left(\bar{y}-\bar{a}_{0}\right)^{n}}{n !} .
$$

Here $\bar{a}_{0}=f(0, \bar{z})$ and is constant, $c_{0}=\bar{c}(0), \bar{c}$ being as in (2.9), and the $b_{n}$ are constants.

The leading-order correction to the basic profile $U_{0}(\bar{y})$ is $O(\bar{x})$ and we find that

$$
\begin{aligned}
\bar{u} & =U_{0}(\bar{y})+\bar{x} \bar{u}_{1}(\bar{y})+o(\bar{x}), \\
v & =\bar{v}_{0}(\bar{y})+\bar{x} \bar{v}_{1}(\bar{y}, \bar{z})+o(\bar{x}), \\
\bar{w} & =\bar{x} \bar{w}_{1}(\bar{y}, \bar{z})+o(\bar{x}), \\
\bar{p}_{\bar{x}} & =p_{0}+o(1), \\
\bar{q} & =q_{0}(\bar{y}, \bar{z})+o(1),
\end{aligned}
$$
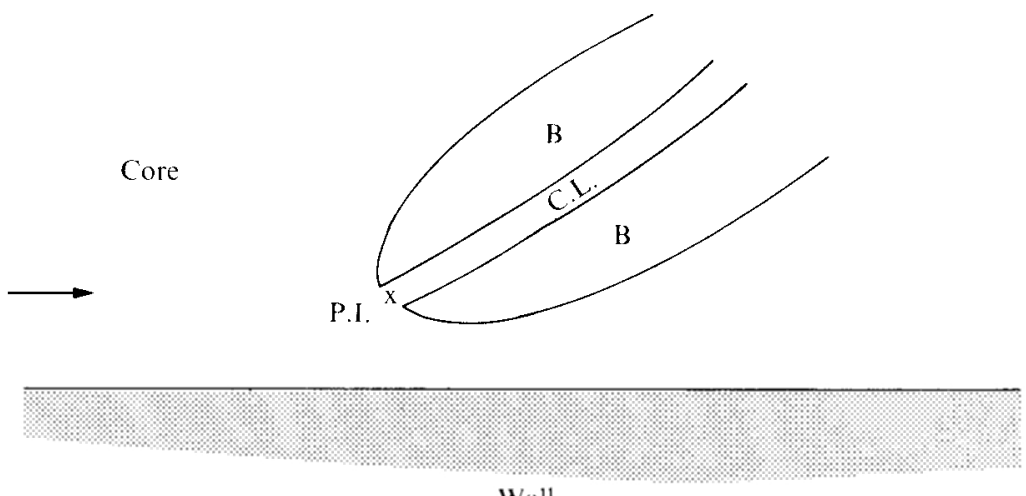

Figure 1. The critical layer C.L. where $\bar{y}-f(\bar{x}, \bar{z})=O\left(R^{-(\delta-h) / 3}\right)$, the buffer layers $\mathrm{B}$, where $\bar{y}-f(\bar{x}, \bar{z})=O\left(\bar{x}^{1 / 2}\right)$, and the core flow at the start of the interaction. P.I. denotes the point of inflection of the oncoming velocity profile. The flow is from left to right. 
where $p_{0}$ is a constant. Equations $(2.8 \mathrm{a}, \mathrm{b})$ then give the solutions

$$
\begin{gathered}
\bar{v}_{0}=U_{0}\left\{\int_{\bar{a}_{0}}^{\bar{y}} \frac{p_{0}-U_{0}^{\prime \prime}}{U_{0}^{2}} d \bar{y}_{1}+\frac{V_{0}}{c_{0}}\right\}, \\
\bar{u}_{1}=\frac{U_{0}^{\prime \prime}-p_{0}}{U_{0}}-U_{0}^{\prime}\left\{\int_{\bar{a}_{0}}^{\bar{y}} \frac{p_{0}-U_{0}^{\prime \prime}}{U_{0}^{2}} d \bar{y}_{1}+\frac{V_{0}}{c_{0}}\right\},
\end{gathered}
$$

where the constant $V_{0}$ is the value of the normal velocity $\bar{v}$ at the initiation of the critical level. We see that these early terms are $\bar{z}$-independent and continuous through $\bar{y}=\bar{a}_{0}$, the continuity of $\bar{v}_{0}$ being determined by the buffer layer discussed below. To effect a match with the buffer layer we shall need the result that, from $(3.3 \mathrm{~b})$, near $\bar{y}=\bar{a}_{0}$,

$$
\bar{u}_{1}(\bar{y})=d_{0}+d_{1}\left(\bar{y}-\bar{a}_{0}\right)+d_{2} \frac{\left(\bar{y}-\bar{a}_{0}\right)^{2}}{2 !}+\ldots,
$$

where

$$
\begin{aligned}
& d_{0}=-\left(p_{0}+V_{0} b_{1}\right) / c_{0}, \quad d_{1}=b_{3} / c_{0}, \\
& d_{2}=\left(b_{4}-V_{0} b_{3}-b_{1} b_{3} / c_{0}\right) / c_{0}, \\
& d_{3}=\left\{b_{5}-b_{4}\left(V_{0}+\frac{2 b_{1}}{c_{0}}\right)+\frac{2 b_{3}}{c_{0}}\left(\frac{b_{1}^{2}}{c_{0}}-p_{0}\right)\right\} / c_{0} .
\end{aligned}
$$

Since the buffer-layer solution of Sections 5 and 6 proceeds in powers of $\bar{x}^{1 / 2}$ the expansions (3.2) will eventually contain powers of $\bar{x}^{1 / 2}$. It is expected that, for example, the leading nonintegral power in $\bar{u}$ is $\bar{x}^{5 / 2}$ and in $\bar{w}$ is $\bar{x}^{3 / 2}$.

\$. The core solution for the wave. We assume the eigensolution of equation (2.9) that comes into existence at $\bar{x}=0$ to have a periodic $\bar{z}$-dependence $\cos \beta_{0} \bar{z}$, and it is the $O(\bar{x})$ correction to the leading harmonic that we shall require. Thus we write

$$
\tilde{p}(\bar{x}, \bar{y}, \bar{z})=\tilde{\pi}_{0}\left\{P_{0}(\bar{y})+\bar{x} P_{1}(\bar{y})\right\} \cos \beta_{0} \bar{z}+O(\bar{x}),
$$

where $O(\bar{x})$ will consist of third order harmonics forced by the buffer layer (see $\$ 6$ ). Also $\tilde{\tilde{\pi}}_{0}$ is a constant, to be determined finally ( $\$ 7$ below). From (2.9) we obtain

$$
\left(U_{0}-c_{0}\right)\left(P_{0}^{\prime \prime}-\gamma_{0}^{2} P_{0}\right)=2 U_{0}^{\prime} P_{0}^{\prime}, \quad P_{0}^{\prime}(0)=P_{0}(\infty)=0,
$$

where $\gamma_{0}^{2}=\alpha_{0}^{2}+\beta_{0}^{2}$ and is determined by this 2D Rayleigh eigenvalue problem. If we normalize $P_{0}$ so that $P_{0}\left(\bar{a}_{0}\right)=1$ then, near $\bar{y}=\bar{a}_{0}$,

$$
P_{0}(\bar{y})=1+\sum_{n=2}^{\infty} \frac{q_{n}\left(\bar{y}-\bar{a}_{0}\right)^{n}}{n !},
$$


where $q_{2}=-\gamma_{0}^{2}, q_{3}$ is not determined by the equation alone (but by its solution), and $q_{4}=-\gamma_{0}^{2}\left(3 \gamma_{0}^{2}+4 b_{3} / b_{1}\right)$.

The equation for $P_{1}(\bar{y})$ obtained at the next order is

$$
\begin{aligned}
& \left(U_{0}-c_{0}\right)\left(P_{1}^{\prime \prime}-\gamma_{0}^{2} P_{1}\right)-2 U_{0}^{\prime} P_{1}^{\prime} \\
& \quad=2 \bar{u}_{1}^{\prime} P_{0}^{\prime}-\left(\bar{u}_{1}-c_{2}\right)\left(P_{0}^{\prime \prime}-\gamma_{0}^{2} P_{0}\right)+2 \alpha_{0} \alpha_{2}\left(U_{0}-c_{0}\right) P_{0},
\end{aligned}
$$

where we have written $\bar{c}(\bar{x})=c_{0}+c_{2} \bar{x}$ and $\alpha(\bar{x})=\alpha_{0}+\alpha_{2} \bar{x}$ correct to $O(\bar{x})$, and the definition of $\bar{c}(\bar{x})$ given following (2.9) implies that $\alpha_{2} c_{0}+\alpha_{0} c_{2}=0$ since the real constant $\Omega$ is specified.

If we denote the right-hand side of equation (4.4) by RHS and write $P_{1}(\bar{y})=P_{0}(\bar{y}) Q_{1}(\bar{y})$ then, for $\bar{y}>\bar{a}_{0}$, we have that

$$
\frac{Q_{1}^{\prime} P_{0}^{2}}{\left(U_{0}-c_{0}\right)^{2}}=\int_{\infty}^{\bar{y}} \frac{(\mathrm{RHS}) P_{0}}{\left(U_{0}-c_{0}\right)^{3}} d \overline{\bar{y}}
$$

while, for $\bar{y}<\bar{a}_{0}$,

$$
\frac{Q_{1}^{\prime} P_{0}^{2}}{\left(U_{0}-c_{0}\right)^{2}}=\int_{0}^{\bar{y}} \frac{(\mathrm{RHS}) P_{0}}{\left(U_{0}-c_{0}\right)^{3}} d \overline{\bar{y}}
$$

on requiring that $P_{1}(\infty)=P_{1}^{\prime}(0)=0$.

The expressions for $P_{1}(\bar{y})$ derived from $(4.5),(4.6)$ above and below the critical level respectively are to be matched to the solutions in the buffer layer. Thus we require the behaviour of the integrands as $\bar{y} \rightarrow \bar{a}_{0} \pm$. It is easily found from (3.1), (3.4) and (4.3) that, near $\bar{y}=\bar{a}_{0}$,

$$
\frac{(\text { RHS }) P_{0}}{\left(U_{0}-c_{0}\right)^{3}}=\frac{a_{-3}}{\left(\bar{y}-\bar{a}_{0}\right)^{3}}+\frac{a_{-2}}{\left(\bar{y}-\bar{a}_{0}\right)^{2}}+\frac{a_{-1}}{\bar{y}-\bar{a}_{0}}+O(1),
$$

where

$$
\begin{aligned}
& a_{-3}=\frac{2 q_{2}}{b_{1}^{3}}\left(c_{2}-d_{0}\right), \\
& a_{-2}=\frac{1}{b_{1}^{3}}\left\{q_{3}\left(c_{2}-d_{0}\right)-2 \alpha_{0}^{2} b_{1} c_{2} / c_{0}\right\}, \\
& a_{-1}=\frac{q_{2}}{b_{1}^{3}}\left\{\frac{c_{2} b_{3}}{b_{1}}-\frac{d_{0} q_{4}}{3 q_{2}}+\frac{d_{0} b_{3}}{3 b_{1}}-d_{0} q_{2}+d_{2}\right\} .
\end{aligned}
$$

As will be seen in Sections 5 and 6 the critical-level conditions (2.14) and the requirement that the solution of (2.9) is regular at $\bar{y}=f(\bar{x}, \bar{z})$, together with the match between the buffer layers and the core, force $a_{-1}$ to be zero. This means that $P_{1}$ contains no logarithmic term at $\bar{a}_{0}$, near which it takes the form

$$
P_{1} \approx b_{1}^{2}\left(\bar{y}-\bar{a}_{0}\right)\left(-\frac{a_{-3}}{2}-\frac{a_{-2}}{2}\left(\bar{y}-\bar{a}_{0}\right)+\frac{1}{3} G^{ \pm}\left(\bar{y}-\bar{a}_{0}\right)^{2}\right)
$$

as $\bar{y} \rightarrow a_{0}^{ \pm}$. Here $G^{ \pm}$are constants whose difference we shall require later. 
It is

$$
\begin{aligned}
G^{+}-G^{-}= & -\int_{0}^{\infty}\left\{\frac{(\mathrm{RHS}) P_{0}}{\left(U_{0}-c_{0}\right)^{3}}-\frac{a_{-3}}{\left(\bar{y}-\bar{a}_{0}\right)^{3}}-\frac{a_{-2}}{\left(\bar{y}-\bar{a}_{0}\right)^{2}}\right\} d \bar{y} \\
& -a_{-3} / 2 \bar{a}_{0}^{2}+a_{-2} / \bar{a}_{0} .
\end{aligned}
$$

A brief summary of the origins of the constants appearing here and in Section 3 might be appropriate here. The properties of the boundary layer that reaches the inflection point at $\bar{x}=0$ are assumed to be known for $\bar{x} \leqslant 0$. Thus $U_{0}(\bar{y})$ is known and hence the constants $b_{n}$ in (3.1). The value of $\bar{y}$ where $U_{0}^{\prime \prime}(\bar{y})=0$ is $\bar{a}_{0}$ and $U_{0}\left(\bar{a}_{0}\right)=c_{0}$. The wave number $\gamma_{0}=\left(\alpha_{0}^{2}+\beta_{0}^{2}\right)^{1 / 2}$ is the eigenvalue of problem (4.2) so, for prescribed $\beta_{0}, \alpha_{0}$ is known as are the constants $q_{n}$ in (4.3). The constants $p_{0}, V_{0}$ in (3.3) will be determined by the boundary conditions at $\bar{y}=\infty$ and the match with a viscous layer on the wall, so $d_{n}$ in (3.5) may be regarded as known. In the following two sections the solutions in the buffer layers will be discussed and matched to those obtained here in the core. The final result of the match will be to determine the constant $\tilde{\pi}_{0}$ in $(4.1)$, but prior to this the match determines the $O(\bar{x})$ perturbation to the position of the critical level (this we denote by $\bar{a}_{2} \bar{x}$ where $\bar{a}_{2}$ turns out to be independent of $\bar{z}$ ), and $c_{2}$ in (4.4), (4.8). For example, if the basic flow happens to be such that $\bar{a}_{2}$ is zero, it emerges that $c_{2}=d_{0}$, and $\bar{u}_{1}\left(\bar{a}_{0}\right) \bar{x}$ at the inner edge of these core regions is also the value of $\bar{u}-c_{0}$ at the critical level $\bar{y}=\bar{a}_{0}$. Again if $\bar{a}_{2}$ happens to be zero then $d_{2}=0$, and $\bar{y}=\bar{a}_{0}$ remains an inflection point of $\bar{u}$ in the core to $O(\bar{x})$. For non-zero $\bar{a}_{2}$, the relations $c_{2}=d_{0}$ and $d_{2}=0$ are modified by a term in $\bar{a}_{2}$ but the above statements regarding the value of $\bar{u}-c_{0}$ at the critical level, now $\bar{y}=\bar{a}_{0}+\bar{a}_{2} \bar{x}$, and the critical level being an inflection point level of the core flow to $O(\bar{x})$ still hold. With $c_{2}=d_{0}$ and $d_{2}=0$ it is easy to verify that $a_{-1}$ in $(4.8 \mathrm{c})$ is zero, and it emerges that this result remains true when these relations are modified by non-zero $\bar{a}_{2}$.

§5. The buffer-layer solution for the vortex. The buffer layer is sandwiched by the core flows of the preceding sections and contains the critical level $\bar{y}=f(\bar{x}, \bar{z})$ as a surface of discontinuity. Across this surface, conditions (2.14) apply and drive the flow. It is convenient to make a Prandtl transformation

$$
\bar{y}-f(\bar{x}, \bar{z})=\bar{Y}, \quad \bar{v}-f_{\bar{x}} \bar{u}-f_{\bar{z}} \bar{w}=\bar{V},
$$

so that equations (2.8) become

$$
\begin{aligned}
& \bar{u}_{\bar{x}}+\bar{V}_{\bar{Y}}+\bar{w}_{\bar{z}}=0 \text {, } \\
& \bar{u} \bar{u}_{\bar{x}}+\bar{V} \bar{u}_{\bar{Y}}+\bar{w} \bar{u}_{\bar{z}}=-\bar{p}_{\bar{x}}+\left(1+f_{\bar{z}}^{2}\right) \bar{u}_{\bar{Y} \bar{Y}}+\bar{u}_{\bar{z} \bar{z}}-f_{\bar{z} \bar{z}} \bar{u}_{\bar{Y}}-2 f_{\bar{z}} \bar{u}_{\bar{Y} \bar{z}}, \\
& \bar{u} \bar{v}_{\bar{x}}+\bar{V} \bar{v}_{\bar{Y}}+\bar{w} \bar{v}_{\bar{z}}=-\bar{q}_{\bar{Y}}+\left(1+f_{\bar{z}}^{2}\right) \bar{v}_{\bar{Y} \bar{Y}}+\bar{v}_{\bar{z} \bar{z}}-f_{\bar{z} \bar{z}} \bar{v}_{\bar{Y}}-2 f_{\bar{z}} \bar{v}_{\bar{Y} \bar{z}}, \\
& \bar{u} \bar{w}_{\bar{x}}+\bar{V} \bar{w}_{\bar{Y}}+\bar{w}_{w_{\bar{z}}}=-\bar{q}_{\bar{z}}+f_{\bar{z}} \bar{q}_{\bar{Y}}+\left(1+f_{\bar{z}}^{2}\right) \bar{w}_{\bar{Y} \bar{Y}}+\bar{w}_{\bar{z} \bar{z}}-f_{\bar{z} \overline{\bar{z}}} \bar{w}_{\bar{Y}}-2 f_{\bar{z}} \bar{w}_{\bar{Y} \overline{\bar{z}}} .
\end{aligned}
$$

At $\bar{Y}=0$ the conditions are now that $\bar{u}, \bar{V}, \bar{w}, \partial \bar{u} / \partial \bar{Y}, \partial \bar{V} / \partial \bar{Y}$ are continuous, and the jump in $\partial \bar{w} / \partial \bar{Y}$ is $J(\bar{x}, \bar{z})$ as in $(2.14)$. 
The solution sought now is in the region $0<\bar{x} \ll 1$ as in Section 3 but now $\bar{Y}=O\left(\bar{x}^{1 / 2}\right)$ so it must match, as $\bar{Y} \bar{x}^{-1 / 2} \rightarrow \pm \infty$, with the solutions of $(3.2)$ as $\bar{y} \rightarrow f(\bar{x}, \bar{z})$.

We attempt a solution of the form

$$
\begin{aligned}
\bar{u} & =c_{0}+b_{1} \bar{Y}+\bar{x} u_{2}(\eta)+\bar{x}^{3 / 2} u_{3}(\eta)+\bar{x}^{2} u_{4}(\eta, \bar{z})+\ldots, \\
\bar{v} & =\frac{1}{c_{0}^{1 / 2}}\left\{v_{0}(\eta)+\bar{x}^{1 / 2} v_{1}(\eta)+\bar{x} v_{2}(\eta, \bar{z})+\ldots\right\}, \\
\bar{w} & =\bar{x}^{1 / 2} w_{1}(\eta, \bar{z})+\ldots, \\
\bar{p}_{\bar{x}} & =p_{0}+p_{2} \bar{x}+\ldots, \\
\bar{q} & =q_{0}(\eta, \bar{z})+\ldots,
\end{aligned}
$$

where

$$
\eta=\frac{\bar{Y}}{2}\left(\frac{c_{0}}{\bar{x}}\right)^{1 / 2}
$$

Also we take

$$
f(\bar{x}, \bar{z})=\bar{a}_{0}+\bar{a}_{2} \bar{x}+O\left(\bar{x}^{2}\right) .
$$

It will be noticed that, except for $\bar{w}$ and $\bar{q}$, the $\bar{z}$-dependence in (5.3) is somewhat "postponed" in the series. For $\bar{u}$ it is $O\left(\bar{x}^{2}\right)$, for $\bar{V}$ it is $O(\bar{x})$ as $\bar{u}$, $\bar{V}$ remain $\bar{z}$-independent until they are affected by the cross-flow $\bar{w}$. Also $b_{1}, p_{0}, p_{2}$ are constants, $b_{1}, p_{0}$ having already appeared in (3.1), (3.2). The constant $\bar{a}_{0}$ is the inflection point of $U_{0}(\bar{y})$ of (3.1), and it will emerge that $\bar{a}_{2}$ in (5.5) is also a constant, the $\bar{z}$-dependence of $f(\bar{x}, \bar{z})$ being deferred at least until the curvature terms.

Equations (5.3)-(5.5) are now substituted into (5.2) and successive powers of $\bar{x}$ collected. We write down only those equations required to determine the terms as far as $u_{4}(\eta, \bar{z})$ as it is $\bar{u}$ that drives the wave pressure $\tilde{p}$ (see (2.9)). The necessary equations derived from the continuity equation (5.2a) are, successively,

$$
\begin{aligned}
v_{0 \eta} & =0 \\
u_{2}-\frac{1}{2} \eta u_{2 \eta}+\frac{1}{2} v_{1 \eta} & =0 \\
\frac{3}{2} u_{3}-\frac{1}{2} \eta u_{3 \eta}+\frac{1}{2} v_{2 \eta}+w_{1 \bar{z}} & =0
\end{aligned}
$$

From the $\bar{x}$-momentum equation ( $5.2 \mathrm{~b})$ we obtain

$$
\begin{gathered}
c_{0}\left(u_{2}-\frac{1}{2} \eta u_{2 \eta}\right)+\mu_{1} v_{0}=-p_{0}+\frac{c_{0}}{4} u_{2 \eta \eta} \\
2 \mu_{1} \eta\left(u_{2}-\frac{1}{2} \eta u_{2 \eta}\right)+c_{0}\left(\frac{3}{2} u_{3}-\frac{1}{2} \eta u_{3 \eta}\right)+\mu_{1} v_{1}+\frac{1}{2} v_{0} u_{2 \eta}=\frac{c_{0}}{4} u_{3 \eta \eta} \\
u_{2}\left(u_{2}-\frac{1}{2} \eta u_{2 \eta}\right)+2 \mu_{1} \eta\left(\frac{3}{2} u_{3}-\frac{1}{2} \eta u_{3 \eta}\right)+c_{0}\left(2 u_{4}-\frac{1}{2} \eta u_{4 \eta}\right)+\mu_{1} v_{2}+\frac{1}{2} v_{1} u_{2 \eta}+\frac{1}{2} v_{0} u_{3 \eta} \\
=-p_{2}+\frac{c_{0}}{4} u_{4 \eta \eta}
\end{gathered}
$$


where we have written

$$
b_{1} / c_{0}^{1 / 2}=\mu_{1}
$$

The leading approximation to the $\bar{Y}$-momentum equation (5.2c) simply repeats (5.6a) and the second approximation determines $q_{0 \eta}$ which we shall not require. However the leading approximation to the $\bar{z}$-momentum equation is important since this marks the first entry of any $\bar{z}$-dependence. It is

$$
w_{1}-\eta w_{1 \eta}=\frac{1}{2} w_{1 \eta \eta} .
$$

The $\bar{z}$-independent terms $u_{2}, u_{3}, v_{0}, v_{1}$ are simply the core solutions in the neighbourhood of $\vec{y}=\vec{a}_{0}$ written in terms of the buffer variables. These are continuous through $\bar{Y}=0$ and are

$$
\begin{aligned}
& v_{0}=c_{0}^{1 / 2}\left(V_{0}-\bar{a}_{2} c_{0}\right), \\
& u_{2}=-\frac{1}{c_{0}}\left[b_{1}\left(V_{0}-\bar{a}_{2} c_{0}\right)+p_{0}\right], \\
& v_{1}=\frac{2 \eta}{c_{0}}\left[b_{1}\left(V_{0}-\bar{a}_{2} c_{0}\right)+p_{0}\right], \\
& u_{3}=\frac{2}{c_{0}^{3 / 2}} b_{3}\left(\frac{2}{3} \eta^{3}+\eta\right) .
\end{aligned}
$$

Here the constant value of $V_{0}$ in $(5.10 \mathrm{a})$ has been chosen to match with the outer solution, the extra term $\bar{a}_{2} c_{0}$ arising from the Prandtl shift and the $c_{0}^{1 / 2}$ factor from the definition of $\bar{v}$ in $(5.3 \mathrm{~b})$. The solutions for $\boldsymbol{u}_{2}, v_{1}, u_{3}$ follow from the respective equations with matching determining the constant in $(5.10 \mathrm{~d})$ to be $b_{3}$. We thus have the value of $c_{2}$ introduced in (4.4) as the $O(\bar{x})$ contribution to the value of $\bar{u}$ at the critical level. It is, from $(5.10 \mathrm{~b})$,

$$
c_{2}=-\frac{1}{c_{0}}\left[b_{1}\left(V_{0}-\bar{a}_{2} c_{0}\right)+p_{0}\right]
$$

because, to this order in $\bar{x}$, the core solution (3.2) holds in the buffer layer as well.

Equation (5.9) must be solved so that $w_{1} \rightarrow 0$ as $|\eta| \rightarrow \infty$, with $w_{1}$ continuous at $\eta=0$ but with a $\bar{z}$-dependent discontinuity in $w_{1 \eta}$. Such a solution is

$$
w_{1}=E_{1}(\bar{z})\left(\eta \operatorname{erf} \eta \mp \eta+\frac{e^{-\eta^{2}}}{\sqrt{ } \pi}\right)
$$

according as $\eta \gtrless 0$. The function $E_{1}(\bar{z})$ will subsequently be related to the jump in $\partial w_{1} / \partial \bar{Y}$ at $\bar{Y}=0$ specified by (2.14).

We continue and find, because $v_{2}$ is forced by $w_{1}$, that

$$
v_{2}=-\frac{2 b_{3}}{c_{0}^{3 / 2}} \eta^{2}-E_{1}^{\prime}(\bar{z})\left(\eta^{2} \operatorname{erf} \eta \mp \eta^{2}+\frac{\eta e^{-\eta^{2}}}{\sqrt{ } \pi}+\frac{1}{2} \operatorname{erf} \eta\right)+A_{2} c_{0}^{1 / 2}
$$

for $\eta \gtrless 0$, where $A_{2}$ is an arbitrary constant whose value is of no consequence 
at this stage. In addition,

$$
\begin{aligned}
u_{4}= & \frac{2}{3} \frac{b_{4}}{c_{0}^{2}} \eta^{4}-\frac{1}{2 c_{0}}\left[c_{2}^{2}+b_{1} A_{2}+p_{2}+\frac{b_{3}}{c_{0}}\left(V_{0}-\bar{a}_{2} c_{0}\right)\right] \\
& -\frac{b_{1}}{3 c_{0}^{3 / 2}} E_{1}^{\prime}(\bar{z})\left[\left(\eta^{4}-\frac{3}{4}\right) \operatorname{erf} \eta+\frac{1}{\sqrt{ } \pi} e^{-\eta^{2}}\left(\eta^{3}-\frac{1}{2} \eta\right) \mp \eta^{4}\right],
\end{aligned}
$$

from $(5.7 \mathrm{c})$. To obtain $u_{4}$ in the form (5.14) various conditions have been satisfied. Firstly, $u_{4}$ and $u_{4 \eta}$ have been made continuous at $\eta=0$. Secondly, the coefficient of $\eta^{4}$ has been chosen to give a match, as $|\eta| \rightarrow \infty$, with the term $b_{4}\left(\bar{y}-\bar{a}_{0}\right)^{4} / 4$ ! in $U_{0}(\bar{y})$ as $\bar{y} \rightarrow \bar{a}_{0}$. Thirdly, we must require that $u_{4 \eta \eta}=0$ at $\eta=0$. The necessity of this condition will be confirmed in Section 6 where it will be seen that it must hold for the solution of equation (6.5) to be regular at $\eta=0$. The condition implies the relation

$$
\frac{b_{4}}{b_{3}}-\frac{b_{1}}{c_{0}}=V_{0}-\bar{a}_{2} c_{0},
$$

which may be regarded as giving $\bar{a}_{2}$, the slope of the critical level $\bar{y}=f(\bar{x}, \bar{z})$ at $\bar{x}=0$, in terms of the given profile $U_{0}(\bar{y})$ and the value of the normal velocity at the initiation of the critical level. A direct consequence of (5.15) is that $a_{-1}$ in $(4.8 \mathrm{c})$ is zero, so that the solution for the wave, $\tilde{p}$, undertaken in Section 4 contains no logarithmic term and continues to be regular. In addition, it is confirmed that $A_{2}$ in (5.13) is independent of $\bar{z}$ since $u_{4}$ at $\eta=0$ must be independent of $\bar{z}$ to be consistent with the initial assumption that $\bar{c}$ is a function of $\bar{x}$ alone.

\$6. The buffer-layer solution for the wave. In this section we obtain the solution for $\tilde{p}$ in (2.9) in the region in which $\eta=O(1)$. The leading terms of the coefficients involving $\bar{u}$ have been obtained in the previous section, and for convenience we first write the equation in terms of $\bar{Y}$ as defined in (5.1). This gives

$$
\begin{aligned}
& (\bar{u}-\bar{c}(\bar{x}))\left[\left(1+f_{\bar{z}}^{2}\right) \tilde{p}_{\bar{Y} \bar{Y}}+\tilde{p}_{\bar{z} \overline{\bar{z}}}-2 f_{\bar{z}} \tilde{p}_{\bar{Y} \overline{\bar{z}}}-f_{\bar{z} \bar{z}} \tilde{p}_{\bar{Y}}-\alpha^{2} \tilde{p}\right] \\
& =2\left[\bar{u}_{\bar{Y}} \tilde{p}_{\bar{Y}}+\left(\tilde{p}_{\bar{z}}-f_{\bar{z}} \tilde{p}_{\bar{Y}}\right)\left(\bar{u}_{\bar{z}}-f_{\bar{z}} \bar{u}_{\bar{Y}}\right)\right],
\end{aligned}
$$

and we set

$$
\begin{aligned}
\tilde{p}= & \pi_{0}(\eta, \bar{z})+\bar{x}_{\pi_{2}}(\eta, \bar{z})+\bar{x}^{3 / 2} \pi_{3}(\eta, \bar{z})+\bar{x}^{2} \pi_{4}(\eta, \bar{z}) \\
& +\bar{x}^{5 / 2} \pi_{5}(\eta, \bar{z})+o\left(\bar{x}^{5 / 2}\right) .
\end{aligned}
$$

It follows successively from (6.1) with (5.3a) and (5.10) that

$$
\begin{aligned}
& \pi_{0}=\Pi_{0}(\bar{z}), \\
& \pi_{2}=\Pi_{2}(\bar{z})+K_{2}(\bar{z}) \eta^{2}, \\
& \pi_{3}=\Pi_{3}(\bar{z})+\tilde{\Pi}_{3}(\bar{z}) \eta^{3}, \\
& \pi_{4}=\Pi_{4}(\bar{z})+\tilde{\Pi}_{4}(\bar{z}) \eta^{3}+K_{4}(\bar{z}) \eta^{4}+L_{4}(\bar{z}) \eta^{2},
\end{aligned}
$$


where

$$
\begin{aligned}
& K_{2}(\bar{z})=\frac{2}{c_{0}}\left(\Pi_{0}^{\prime \prime}-\alpha_{0}^{2} \Pi_{0}\right), \\
& K_{4}(\bar{z})=\frac{8}{3} \frac{b_{3}}{b_{1} c_{0}^{2}}\left(\Pi_{0}^{\prime \prime}-\alpha_{0}^{2} \Pi_{0}\right)-\frac{1}{c_{0}}\left(K_{2}^{\prime \prime}-\alpha_{0}^{2} K_{2}\right), \\
& L_{4}(\bar{z})=\frac{2}{c_{0}}\left(\Pi_{2}^{\prime \prime}-\alpha_{0}^{2} \Pi_{2}-2 \alpha_{0} \alpha_{2} \Pi_{0}\right) .
\end{aligned}
$$

Here $\Pi(\bar{z}), \tilde{\Pi}(\bar{z})$ are, at present, arbitrary functions and we shall subsequently take $\Pi_{0}(\bar{z})=\tilde{\tilde{\pi}}_{0} \cos \beta_{0} \bar{z}$ in order to achieve a match with (4.1).

The equation for $\pi_{5}(\eta, \bar{z})$ is more complicated, since it is partly forced by $\bar{u}_{4}$ in $(5.3 \mathrm{a})$. It is, upon substitution for $\bar{u}_{2}, \bar{u}_{3}$,

$$
\begin{aligned}
& \frac{c_{0}}{4}\left(\eta \frac{\partial^{2} \pi_{5}}{\partial \eta^{2}}-\frac{2 \partial \pi_{5}}{\partial \eta}\right) \\
& =\frac{2 b_{3}}{b_{1}} \eta^{4} \tilde{\Pi}_{3}-\left(\Pi_{3}^{\prime \prime}+\tilde{\Pi}_{3}^{\prime \prime} \eta^{3}\right) \eta+\alpha_{0}^{2}\left(\Pi_{3}+\tilde{\Pi}_{3} \eta^{3}\right) \eta \\
& \quad-c_{0}^{1 / 2}\left\{\bar{a}_{4}^{\prime} \Pi_{0}^{\prime}+\frac{1}{b_{1}}\left(\Pi_{0}^{\prime \prime}-\alpha_{0}^{2} \Pi_{0}\right)\left(u_{4}-c_{4}-\eta \frac{\partial u_{4}}{\partial \eta}\right)-\frac{1}{b_{1}} \Pi_{0}^{\prime} \frac{\partial u_{4}}{\partial \bar{z}}\right\} .
\end{aligned}
$$

The vanishing of $u_{4 \eta \eta}$ at $\eta=0$ ensures, as explained above (5.15), the absence of a term in $\pi_{5}$ proportion to $\eta^{2} \log |\eta|$ for $|\eta| \ll 1$. In $(6.5), \bar{a}_{4}(\bar{z})$ is the term $O\left(\bar{x}^{2}\right)$ in $f(\bar{x}, \bar{z})$; its presence is of no consequence.

Our sole requirement from the solution of the equation for $\pi_{5}$ is the difference in the coefficients of $\eta^{3}$ in $\pi_{5}$ as $\eta \rightarrow \pm \infty$. It is clear that only $u_{4}$ contributes to this and indeed only the terms multiplying $E_{1}^{\prime}(\vec{z})$ in $(5.14)$. It is easily found that this difference is

$$
-\frac{4}{3 c_{0}^{2}}\left\{E_{1}^{\prime}(\bar{z})\left(\Pi_{0}^{\prime \prime}-\alpha_{0}^{2} \Pi_{0}\right)+E_{1}^{\prime \prime}(\bar{z}) \Pi_{0}^{\prime}\right\} .
$$

We have not yet used the jump condition (2.14) which relates $E_{1}(\bar{z})$ to $\Pi_{0}\left(=\tilde{\pi}_{0} \cos \beta_{0} \bar{z}\right)$. To leading order in $\bar{x}(2.14)$ reduces to

$$
\left[w_{1 \eta}\right]_{-}^{+}=\frac{4 \pi\left(\frac{2}{3}\right)^{2 / 3}\left(-\frac{2}{3}\right) !}{c_{0}^{1 / 2}\left(\alpha_{0} b_{1}\right)^{5 / 3}} \frac{\partial}{\partial \bar{z}}\left\{\left|\tilde{p}_{\bar{z}}\right|^{2}\right\}
$$

and (5.12) shows that the value of the left-hand side is $-2 E_{1}(\bar{z})$. This enables us to identify the term in $\cos \beta_{0} \bar{z}$ in (6.6) as

$$
\frac{8 \pi}{3 c_{0}^{5 / 2}} \frac{\left(\frac{2}{3}\right)^{2 / 3}\left(-\frac{2}{3}\right) !}{\left(\alpha_{0} b_{1}\right)^{5 / 3}} \beta_{0}^{4}\left(\beta_{0}^{2}-\alpha_{0}^{2}\right) \tilde{\pi}_{0}\left|\tilde{\pi}_{0}\right|^{2} \cos \beta_{0} \bar{z} .
$$

The equation satisfied by the constant $\tilde{\pi}_{0}$ now emerges from the match between the difference in the coefficients of $\bar{x} \bar{y}^{3}$ as $\bar{y} \rightarrow \bar{a}_{0} \pm$ in the core solution for $\tilde{p}$ in Section 4, and the difference in the coefficients of $\eta^{3}$ as $\eta \rightarrow \pm \infty$ as obtained in this section. The expression (6.6) forces higher harmonics of $O(\bar{x})$ on the core solution (4.1) but it is the term in $\cos \beta_{0} \bar{z}$ that leads to the value of $\left|\tilde{\pi}_{0}\right|^{2}$. The result is given in the following section. 
\$7. The equation satisfied by $\tilde{\tilde{\pi}}_{0}$. It remains to match the term in (4.1) that is $O\left\{\bar{x}\left(\bar{y}-\bar{a}_{0}\right)^{3} \cos \beta_{0} \bar{z}\right\}$ as $\bar{y} \rightarrow \bar{a}_{0} \pm$ with the contribution to (6.2) that is $O\left(\bar{x}^{5 / 2} \eta^{3} \cos \beta_{0} \bar{z}\right\}$ as $\eta \rightarrow \pm \infty$. It is the differences of these terms that lead to the value of $\tilde{\tilde{\pi}}_{0}$, and from (4.9), (4.10) and (6.8) we obtain

$$
\begin{aligned}
& \frac{\left(\frac{2}{3}\right)^{2 / 3}\left(-\frac{2}{3}\right) !}{c_{0}\left(\alpha_{0} b_{1}\right)^{5 / 3}} \pi \beta_{0}^{4}\left(\alpha_{0}^{2}-\beta_{0}^{2}\right) \tilde{\pi}_{0}\left|\tilde{\pi}_{0}\right|^{2} \\
& \quad=\tilde{\pi}_{0} b_{1}^{2}\left[\int_{0}^{\infty}\left\{\frac{(\mathrm{RHS}) P_{0}}{\left(U_{0}-c_{0}\right)^{3}}-\frac{a_{-3}}{\left(\bar{y}-\bar{a}_{0}\right)^{3}}-\frac{a_{-2}}{\left(\bar{y}-\bar{a}_{0}\right)^{2}}\right\} d \bar{y}+\frac{a_{-3}}{2 \bar{a}_{0}^{2}}-\frac{a_{-2}}{\bar{a}_{0}}\right] .
\end{aligned}
$$

The solution $\tilde{\tilde{\pi}}_{0}=0$ corresponds to the zero-interaction situation with no wave but it seems highly likely that a positive value of $\left|\tilde{\tilde{\pi}}_{0}\right|^{2}$ can be obtained by suitable choice of $\alpha_{0}^{2}$, thus allowing a bifurcation. This possibility is examined in the following section. The sum of $\alpha_{0}^{2}+\beta_{0}^{2}\left(=\gamma_{0}^{2}\right)$ is not, of course, at our disposal since it is the eigenvalue of the original problem for $P_{0}(\bar{y})$ in (4.2).

\$8. A representative basic flow. We now demonstrate explicitly the existence of a non-zero $\tilde{\pi}_{0}$ in (7.1) for a representative basic flow. The flow chosen is a modification of the model velocity profile proposed by Smith and Bodonyi (1985). For simplicity we consider the non-interactive case and take $U_{0}(\bar{y})$ in $(4.2)$ to be the solution of

$$
\frac{U_{0 \bar{y} \bar{y}}}{U_{0}-c_{0}}=-h e^{-2 \bar{y}}+h^{2} \mu^{2} e^{-4 \bar{y}},
$$

with $U_{0}(0)=0, U_{0}(\infty)=1$. Here, as in Smith and Bodonyi, the right-hand side, with constant $h$ and $\mu$, is chosen to be exponentially small as $\bar{y} \rightarrow \infty$, and to be such that the left-hand side leads to a regular, inflectional, critical layer. The additional parameter $\mu$ in (8.1) will permit us to choose $U_{0}^{\prime \prime \prime}(0)=0$, a requirement that is necessary to prevent the occurrence of a term $O(\bar{x} \log \bar{x})$ in the core flow (3.2a), a complication that is not permitted in the present theory.

With

$$
\tilde{z}=\sqrt{ } h e^{-\bar{y}} \quad \text { and } \quad \frac{U_{0}-c_{0}}{1-c_{0}}=S(\tilde{z}),
$$

the equation satisfied by $S(\tilde{z})$ is

$$
\tilde{z}^{2} S^{\prime \prime}+\tilde{z} S^{\prime}+\left(\tilde{z}^{2}-\mu^{2} \tilde{z}^{4}\right) S=0,
$$

with solution regular at $\tilde{z}=0$

$$
S(\tilde{z})=e^{-\mu \tilde{z}^{2} / 2} M\left(\frac{1}{2}-\frac{1}{4 \mu}, 1, \mu \tilde{z}^{2}\right),
$$

where $M(a, b, s)$ is the confluent hypergeometric function in the usual notation. The corresponding solution for $P_{0}$ in (4.2) is then

$$
P_{0}(\bar{y})=A_{0} \tilde{z}\left(\psi S^{\prime}-S \psi^{\prime}\right),
$$


where the primes in (8.5) denote differentiation with respect to $\tilde{z}$ and

$$
\psi(\tilde{z})=\tilde{z}^{\gamma_{0}} e^{-\mu \tilde{z}^{2} / 2} M\left(\frac{1}{2}+\frac{1}{2} \gamma_{0}-\frac{1}{4 \mu}, 1+\gamma_{0}, \mu \tilde{z}^{2}\right) .
$$

The constant $\boldsymbol{A}_{0}$ is to be chosen so that $P_{0}$ is unity where $U_{0}=c_{0}$ as in (4.3). The solutions (8.2), (8.5) satisfy the conditions at $\bar{y}=\infty(\tilde{z}=0)$, and now the conditions at the wall $\tilde{z}=\sqrt{ } h$ where $U_{0}(\bar{y})=P_{0}^{\prime}(\bar{y})=0$ (and hence $\left.\psi(\sqrt{ } h)=0\right)$, and at the critical level $\bar{y}=\bar{a}_{0}\left(\tilde{z}=\tilde{z}_{c}\right)$ where $S=0$ and $P_{0}=1$, must be considered.

We first choose $U_{0}^{\prime \prime \prime}(0)=0$ so that $\bar{u}_{1}$ in $(3.3 \mathrm{~b})$ contains no term in $\log \bar{y}$ as $\bar{y} \rightarrow 0$. The requirement is equivalent to choosing $h$ in terms of $\mu$ so that

$$
\sqrt{ } h \frac{S^{\prime}(\sqrt{ } h)}{S(\sqrt{ } h)}=\frac{2\left(2 \mu^{2} h-1\right)}{1-\mu^{2} h} .
$$

Also the resulting profile must have no back-flow, for if $U_{0}(\bar{y})$ has an additional zero for $\bar{y}>0$, the profiles would have to satisfy additional compatability conditions there in order for $\bar{u}_{1}$ in $(3.3 \mathrm{~b})$ to be sufficiently regular for the theory of the preceding sections to be valid. The simple right-hand side of (8.1) does not permit such extra conditions to be imposed. In fact the only possible profile is monotonic with $S(0)=1, S\left(\tilde{z}_{c}\right)=0, S(\sqrt{ } h)<0$ and $S^{\prime}(\sqrt{ } h)<0$. We see from (8.7) that $\frac{1}{2} \leqslant \mu^{2} h \leqslant 1$ and it is not difficult to interpret the limiting cases $\mu^{2} h=\frac{1}{2}$ and $\mu^{2} h=1$ as respectively corresponding to a separated profile and one for which the critical layer is on the wall. For the former

$$
\mu=\frac{1}{6}, \quad \sqrt{ } h=3 \sqrt{ } 2 \quad \text { and } \quad S(\tilde{z})=e^{-\tilde{z}^{2} / 12}\left(1-\tilde{z}^{2} / 6\right)
$$

so that $\tilde{z}_{c}=\sqrt{ } 6, \tilde{a}_{0}=\frac{1}{2} \log 3$ and $c_{0}=2 /\left(2+e^{3 / 2}\right)$. For the latter the corresponding parameters have to be determined numerically and are

$$
\mu=0 \cdot 3698, \quad \sqrt{ } h=2 \cdot 7044,
$$

with $\tilde{z}_{c}=\sqrt{ } h$ and $c_{0}=0$. Although these limiting cases, particularly the latter, are of interest in themselves they will not be considered here.

Once $h$ has been determined as a function of $\mu$ (for $\frac{1}{6}<\mu<0.3968$ ), the value of $\gamma_{0}$ is determined so that $P_{0}^{\prime}(\bar{y})$ calculated from (8.5) vanishes at $\tilde{z}=\sqrt{ } h$. For the limiting cases above, $\gamma_{0}=1.0352$ and $\gamma_{0}=0$ respectively. For convenience we restrict ourselves to the two values of $\mu$ which respectively give rise to the values $\gamma_{0}=1.0$ and $\gamma_{0}=0.5$ and shall find that in both these cases equation (7.1) has an acceptable solution for $\tilde{\tilde{\pi}}_{0}^{2}$.

Now that $\gamma_{0}, \sqrt{ } h, \mu$ have been decided upon, all the quantities required in (7.1) are formally determined except for $\alpha_{0}$ and $\tilde{\pi}_{0}^{2}$ which the equation relates. In $(3.3 \mathrm{~b})$ we choose $p_{0}=U_{0}^{\prime \prime}(0)$ and $V_{0}$ so that $\bar{u}_{1}$ vanishes at $\bar{y}=0$. Thus the core flow (3.2a) vanishes on the wall to $O(\bar{x})$ and any singularity is, as is to be expected, situated away from the wall at the initiation of the critical level. The principal results of the numerical integration to determine the integrals in (7.1) are summarized below.

Case (a) $\gamma_{0}=1 \cdot 0$.

For this $\mu=0 \cdot 1716, \sqrt{ } h=4 \cdot 2032, c_{0}=0.3104, \tilde{z}_{c}=2.4524 \quad\left(\bar{a}_{0}=0.5388\right)$, 
$b_{1}=0 \cdot 8350, b_{3}=-4 \cdot 1318$, and $(7.1)$ becomes

$$
\frac{Q_{0}^{2}}{2858 \cdot 8}=\frac{\left(1+0 \cdot 0356 \alpha_{0}^{2}\right) \alpha_{0}^{5 / 3}}{\left(1-\alpha_{0}^{2}\right)^{2}\left(2 \alpha_{0}^{2}-1\right)},
$$

where

$$
Q_{0}^{2}=\left(\frac{2}{3}\right)^{2 / 3}\left(-\frac{2}{3}\right) ! \pi\left|\tilde{\pi}_{0}\right|^{2} / c_{0} b_{1}^{11 / 3} .
$$

It is clear that (8.10) has an acceptable value for $Q_{0}^{2}$ for $\frac{1}{2}<\alpha_{0}^{2}<1$.

Case (b) $\gamma_{0}=0 \cdot 5$.

For this $\mu=0.2387, \sqrt{ } h=3.4901, c_{0}=0.2643, \tilde{z}_{c}=2.5029 \quad\left(\bar{a}_{0}=0.3325\right)$, $b_{1}=0 \cdot 8614, b_{3}=-3 \cdot 4708$, and (7.1) becomes

$$
\frac{Q_{0}^{2}}{118 \cdot 36}=\frac{\left(1+1 \cdot 8452 \alpha_{0}^{2}\right) \alpha_{0}^{5 / 3}}{\left(\frac{1}{4}-\alpha_{0}^{2}\right)^{2}\left(2 \alpha_{0}^{2}-\frac{1}{4}\right)},
$$

$Q_{0}^{2}$ again being given by (8.11). This time we require $\frac{1}{8}<\alpha_{0}^{2}<\frac{1}{4}$. The values of the integrals required in (7.1) were confirmed, where necessary, by integration in the complex plane. Profiles of $U_{0}(\bar{y})$ plotted both as a function of $\tilde{z}$ and of $\bar{y}$ are shown in Figs. 2 and 3 for the values $\mu=0 \cdot 17, \mu=0 \cdot 24$. In all possible cases the critical layer is very close to the wall, its $\bar{y}$ position varying from zero when $\mu=0.37$ to 0.549 where $\mu=\frac{1}{6}$.

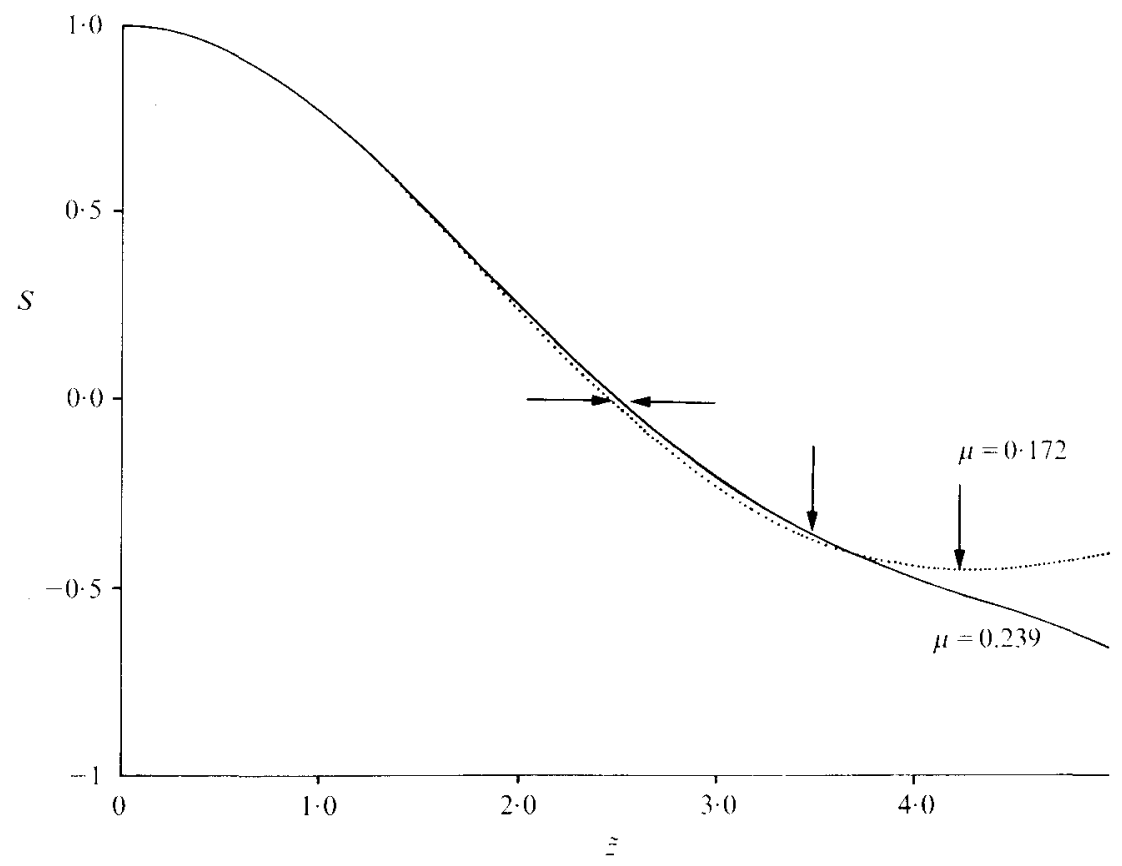

Figure 2. Plot of $S$ against $\tilde{z}$ for $\mu=0 \cdot 172$ and $\mu=0 \cdot 239$. The horizontal arrows denote the position of the critical layer (where $S$ is zero) and the vertical arrows that of the wall (where $U_{0}$ is zero). 


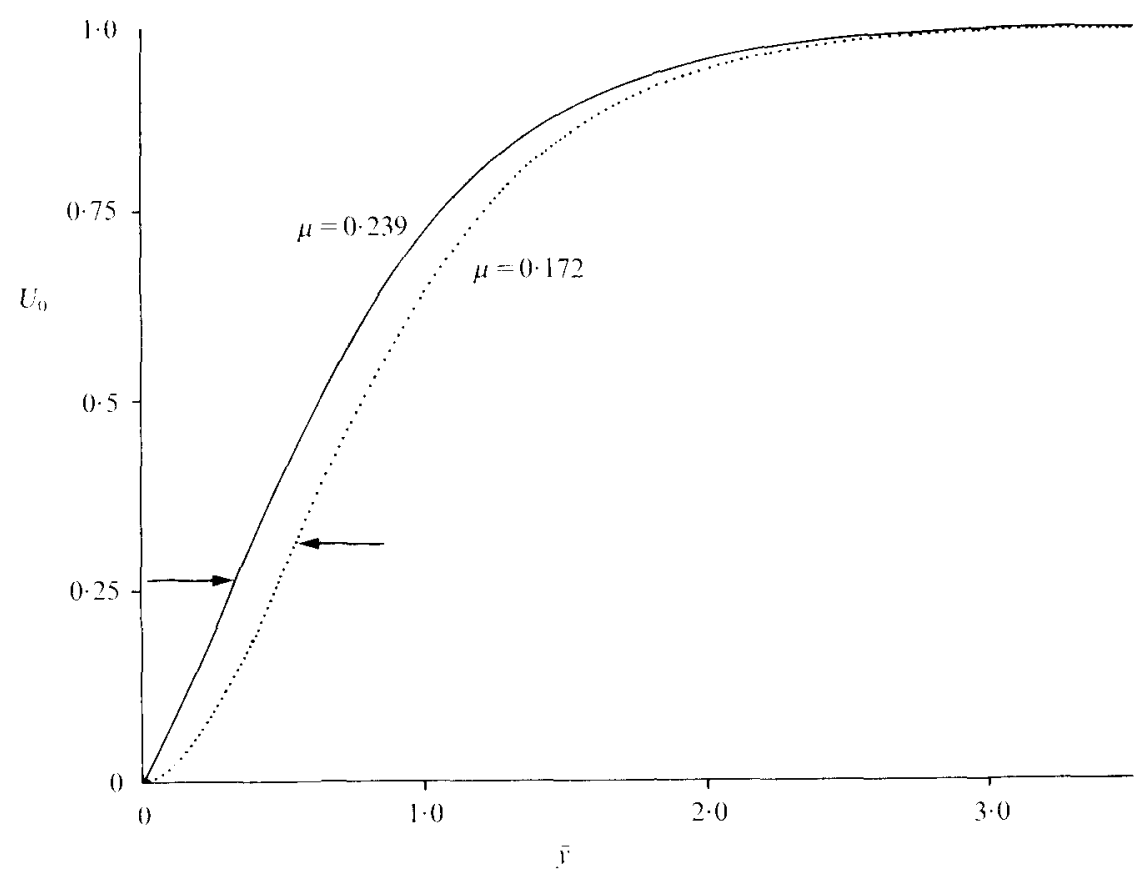

Figure 3. Plot of $U_{0}$ against $\bar{y}$ for $\mu=0 \cdot 172$ and $\mu=0 \cdot 239$. The horizontal arrows denote the position of the critical layer (at the point of inflection).

It seems likely that, in the acceptable range of $\mu\left(\frac{1}{6}<\mu<0 \cdot 3698\right)$, equation (7.1) leads to positive values for $Q_{0}^{2}$ and $\alpha_{0}^{2}$ for a range of $\alpha_{0}^{2}$ with $\frac{1}{2} \gamma_{0}^{2}<\alpha_{0}^{2}<\gamma_{0}^{2}$ although we have confirmed this result for $\gamma_{0}=\frac{1}{2}, 1$ only. Thus for infinitely long waves the solution will exist only when the critical layer is on the wall, and the largest range of wavenumbers is possible for the separating profile. Although these conclusions are interesting it must be remembered that this deduction has been made by using the relatively simple respresentative velocity profiles generated by $(8.1)$.

\$. Further comments. The existence of a solution as suggested by Section 8 , to start the vortex/Rayleigh-wave interaction, is a helpful point for a number of reasons. It does not imply that the solution continues for all positive $\bar{x}$, of course, and ideas on the terminal behaviour of vortex/wave interactions further downstream are discussed by Smith and Walton (1989), Hall and Smith (1991), Walton and Smith (1992). It could however be used, first, to compare with direct computational results. This is especially in view of the multi-structured form of the interaction at the start (Sections 3-7) which would be difficult to handle accurately in a standard numerical treatment. Another use of the analysis for the present starting response is in pointing to the different interactions that can occur on shorter length scales such as those considered by Davis (1992) and Smith et al. (1992). 
The analysis of the present paper can be formally extended to incorporate higher approximations of the asymptotic solution at small $\bar{x}$. A brief discussion of the flow in the buffer zone is given here.

The next approximation for the vortex flow expansions (5.5) to (5.7) contains the terms $\bar{x}^{5 / 2} u_{5}(\eta, \bar{z}), c_{0}^{-1 / 2} \bar{x}^{3 / 2} v_{3}(\eta, \bar{z}), \bar{x} w_{2}(\eta, \bar{z})$ respectively. Also the term $\bar{x}^{3} \pi_{6}(\eta, \bar{z})$ must be added in the expansion for the wave pressure (6.2). The governing equation for the wave term $\pi_{6}$ is similar to (6.5), with $\pi_{5}$ replaced by $\pi_{6}$ and a more lengthy expression on the right-hand side. The requirement for $\pi_{6}$ to be regular at the critical surface provides a restriction on the curvature of the velocity profile at $\eta=0$, namely

$$
\left.\frac{\partial^{2} u_{5}}{\partial \eta^{2}}\right|_{\eta=0}=0 .
$$

The same condition for the function $u_{4}(\eta, \bar{z})$ was derived in $\$ 5$ (and we also note that the first term with nonzero curvature at $\eta=0$ is of the order $O\left(\bar{x}^{3}\right)$ in (5.3a), owing to the three-dimensional shape of the critical layer).

The condition (9.1) is essential to the derivation of the vortex-flow functions $u_{5}, v_{3}, w_{2}$, as can be seen from the symmetry properties of the flow field in the buffer. The leading three-dimensional terms for the vortex $u_{4}, v_{2}, w_{2}$ are governed by the balancing

$$
\bar{V}_{\bar{Y}}+\bar{w}_{\bar{z}}=0, \quad c_{0} \bar{u}_{\bar{x}}+b_{1} \bar{V}=\bar{u}_{\bar{Y} \bar{Y}}, \quad c_{0} \bar{w}_{\bar{x}}=\bar{w}_{\bar{Y} \bar{Y}},
$$

which follow from equation $(5.2 \mathrm{a}, \mathrm{b}, \mathrm{d})$ respectively. With the jump in $\partial \bar{w} / \partial \bar{Y}$ and the smoothness conditions imposed on the other functions, as discussed in $\$ 2$, one can easily deduce from (9.2) that $w_{2}$ is an even, while $u_{4}$ is an odd, function of $\eta$. The latter property in fact leads to the solution for $u_{4}$ which satisfies a condition of the form (9.1).

In the next approximation, however, the functions $w_{2}, u_{5}$ contain both even and odd constituents due to the nonuniform terms entering the governing equations. For instance, the derivatives $v_{0} \partial w_{1} / \partial \eta, v_{0} \partial u_{4} / \partial \eta$ appear as forcing terms in the momentum equations for $w_{2}, u_{5}$ respectively. The constraint (9.1) now provides an extra boundary condition for the vortical flow, which cannot be satisfied in the case of a general velocity profile $U_{0}(\bar{y})$. Omitting details of calculation we state here the solvability condition for the corresponding boundary-value problem in the form

$$
\frac{b_{4}}{b_{3}}-\frac{5}{2} \frac{b_{1}}{c_{0}}=0
$$

relating the higher-order Taylor coefficients of the unperturbed velocity profile (3.1) at the start of the vortex-wave interaction.

Further analysis is necessary to establish whether solvability at the higher approximations imposes additional restrictions on the shape of the unperturbed velocity profile. However, we believe that, regardless of the number and the particular form of the solvability conditions, the class of physically realistic flows is wide enough to provide velocity fields which satisfy all the necessary constraints. This is because the local Taylor coefficients involved depend on the global evolution of the flow upstream and hence can be easily altered at 
any given $\bar{x}$-section of the boundary layer. On the other hand, the existence of the solvability conditions indicates the strong sensitivity of the solution obtained to the properties of the unperturbed flow. This in turn suggests the possibility of alternative routes for the flow behaviour after the start of a strong vortex-wave coupling on a shorter length scale.

The current work is for an incompressible boundary layer with a twodimensional starting profile. Alternatively the vortex/Rayleigh wave interaction may perhaps start following a vortex/Tollmien-Schlichting-wave interaction upstream (see the papers mentioned above), for example, involving a three-dimensional starting profile. Again, the compressible version could well yield equally interesting features. There are numerous other applications also.

The repercussion that seems foremost to us now however concerns the flow on a shorter length scale. There the core-plus-buffer flow structure and singularities studied in Sections 3-7 must be smoothed out, by a new physical effect or effects, possibly allowing the flow solution to be joined smoothly to that upstream as opposed to the abrupt departure involved in Sections 3-7. The new flow structure is a rather complex one, studied by Smith et al. (1992), and is inferred directly from the present work.

Thanks are due to S.E.R.C. (for P.G.B.'s financial support and for computing), to A.F.O.S.R. (for F.T.S.), to United Technologies and the Royal Society (for S.N.T.'s financial support), and to Dr. D. A. R. Davis and Professor M. R. Foster for related discussions.

Appendix. More details of the critical-layer solution. We now illustrate the derivation of (2.14) by considering the solution in the neighbourhood of the critical level. Here $\bar{x}=O(1)$ and the expansions are made in suitable inverse powers of the Reynolds number. The width of the critical layer is $O\left(R^{-(\delta-b) / 3}\right)$ and we therefore define

$$
\bar{y}-f(\bar{x}, \bar{z})=R^{-2 \sigma} Y_{1}, \quad \sigma=\frac{1}{6}(\delta-b) .
$$

Consideration of the forms of $(2.7)$ as $\bar{y} \rightarrow f(\bar{x}, \bar{z})$ suggests that we write

$$
\left.\begin{array}{rl}
u & =R^{-b-1+2 \delta}\left\{\bar{c}(\bar{x})+R^{-2 \sigma} \bar{\lambda}(\bar{x}, \bar{z}) Y_{1}+R^{-4 \sigma} \bar{u}_{0}+R^{-5 \sigma} \bar{u}_{1}+R^{-6 \sigma} \bar{u}_{2}+\ldots\right\}, \\
v & =R^{-1+\delta}\left\{R^{\sigma} \bar{v}_{1}+\bar{v}_{2}+R^{-\sigma} \bar{v}_{3}+\ldots\right\}, \\
w & =R^{-1+\delta}\left\{R^{\sigma} \bar{w}_{1}+\bar{w}_{2}+R^{-\sigma} \bar{w}_{3}+\ldots\right\}, \\
p & =R^{-2 b-2+4 \delta}\left\{\bar{p}(\bar{x})+R^{-7 \sigma} \bar{p}_{1}+R^{-9 \sigma} \bar{p}_{3}+R^{-11 \sigma} \bar{p}_{5}+R^{-12 \sigma} \bar{p}_{6}+\ldots\right\},
\end{array}\right\}
$$

the powers $R^{-5 \sigma}$ in $\bar{u}_{1}$ and $R^{\sigma}$ in $\bar{v}_{1}, \bar{w}_{1}$ occurring because $\tilde{u}, \tilde{v}, \tilde{w}$ in (2.7) have a factor $(\bar{y}-f(\bar{x}, \bar{z}))^{-1}$ as $\bar{y} \rightarrow f(\bar{x}, \bar{z})$ although $\tilde{p}$ is regular. It emerges that $\bar{p}_{2}=\bar{p}_{4}=0$ so these terms have been omitted from the expansion for $p$. The nature of these terms is also fairly readily ascertained from the equations they satisfy and from the required match with (2.7). We deduce that $\bar{u}_{1}, \bar{v}_{1}, \bar{w}_{1}, \bar{p}_{1}$ have a term multiplying $E$ and one in $E^{-1}$ (as in (2.4)) but no $E$-independent term, and that $\bar{u}_{3}, \bar{v}_{3}, \bar{w}_{3}, \bar{p}_{3}, \bar{p}_{5}$ are also of this form. The terms with suffix two are $E$-independent, and are simply the appropriate contribution from the outer solution, namely $\bar{v}_{2}=\bar{v}(\bar{x}, f(\bar{x}, \bar{z}), \bar{z})$ and $\bar{w}_{2}=\bar{w}(\bar{x}, f(\bar{x}, \bar{z}), \bar{z})$ with 
$\bar{u}_{2} \propto Y_{1}^{3}$. The important equations are those for the terms $\bar{v}_{4}, \bar{w}_{4}, \bar{p}_{6}$ which are made up of terms in $E^{ \pm 1}, E^{ \pm 2}$ and $E$-independent terms, the last of which, on matching with (2.7), supply conditions (2.14).

We return to the full incompressible Navier-Stokes equations, making the scaling (2.3) and the transformation (A1) and writing

$$
\begin{aligned}
& \frac{\partial}{\partial x}=R^{\delta} \frac{\alpha(\bar{x})}{\alpha_{0}} \frac{\partial}{\partial X}+R^{b}\left(\frac{\partial}{\partial \bar{x}}-R^{2 \sigma} f_{\bar{x}} \frac{\partial}{\partial Y_{1}}\right), \\
& \frac{\partial}{\partial z}=R^{\delta}\left(\frac{\partial}{\partial \bar{z}}-R^{2 \sigma} f_{\bar{z}} \frac{\partial}{\partial Y_{1}}\right),
\end{aligned}
$$

where $X$ is defined in (2.5). Thus (A4) accommodates a Prandtl origin shift. The required equations are then, firstly from the $x$-momentum component,

$$
\begin{gathered}
\bar{v}_{1}-f_{\bar{z}} \bar{w}_{1}=0, \\
\bar{c} \bar{c}^{\prime}-\bar{c} f_{\bar{x}} \bar{\lambda}+\bar{\lambda}\left(\bar{v}_{2}-f_{\bar{z}} \bar{w}_{2}\right)=-\bar{p}^{\prime}(\bar{x})-f_{\bar{z}} \lambda_{\bar{z}}-\frac{\partial}{\partial \bar{z}}\left(f_{\bar{z}} \bar{\lambda}\right)+\Delta \frac{\partial^{2} \bar{u}_{0}}{\partial Y_{1}^{2}}, \\
\frac{\alpha}{\alpha_{0}} \bar{\lambda} Y_{1} \frac{\partial \bar{u}_{1}}{\partial X}+\bar{\lambda}\left(\bar{v}_{3}-f_{\bar{z}} \bar{w}_{3}\right)+\lambda_{\bar{z}} Y_{1} \bar{w}_{1}=-\frac{\alpha}{\alpha_{0}} \frac{\partial \bar{p}_{1}}{\partial X}+\Delta \frac{\partial^{2} \bar{u}_{1}}{\partial Y_{1}^{2}},
\end{gathered}
$$

where $\Delta=1+f_{\bar{z}}^{2}$ as in (2.14). From the $\bar{y}$ - and $\bar{z}$-momentum equations we first deduce that $\bar{p}_{1}$ is independent of $Y_{1}$ so that

$$
\bar{p}_{1}=\tilde{p}(\bar{x}, f(\bar{x}, \bar{z}), \bar{z}) E+\text { c.c. }
$$

on matching with $(2.7 \mathrm{c})$. We also find that $\bar{v}_{2}, \bar{w}_{2}$ are each independent of $Y_{1}$ and, from (A5b), that $\bar{u}_{0} \propto Y_{1}^{2}$ for $Y_{1} \gg 1$. This demonstrates how the curvature of the streamwise profile develops with $\bar{x}$ and $\bar{z}$ as it becomes non-inflectional. Because we do not require $\bar{v}_{3}, \bar{w}_{3}, \bar{p}_{5}$ independently it is sufficient to retain the equations

$$
\begin{gathered}
\frac{\alpha}{\alpha_{0}} \bar{\lambda} Y_{1} \frac{\partial \bar{v}_{1}}{\partial X}=-\frac{\partial \bar{p}_{3}}{\partial Y_{1}}+\Delta \frac{\partial^{2} \bar{v}_{1}}{\partial Y_{1}^{2}} \\
\frac{\alpha}{\alpha_{0}} \bar{\lambda} Y_{1} \frac{\partial \bar{v}_{4}}{\partial X}+\frac{\alpha}{\alpha_{0}} \bar{u}_{1} \frac{\partial \bar{v}_{1}}{\partial X}+\left(\bar{v}_{3}-f_{\bar{z}} \bar{w}_{3}\right) \frac{\partial \bar{v}_{1}}{\partial Y_{1}}+\bar{w}_{1} \frac{\partial \bar{v}_{1}}{\partial \bar{z}}=-\frac{\partial \bar{p}_{6}}{\partial Y_{1}}+\Delta \frac{\partial^{2} \bar{v}_{4}}{\partial Y_{1}^{2}},
\end{gathered}
$$

and

$$
\begin{gathered}
\frac{\alpha}{\alpha_{0}} \bar{\lambda} Y_{1} \frac{\partial \bar{w}_{1}}{\partial X}=f_{\bar{z}} \frac{\partial \bar{p}_{3}}{\partial Y_{1}}-\frac{\partial \bar{p}_{1}}{\partial \bar{z}}+\Delta \frac{\partial^{2} \bar{w}_{1}}{\partial Y_{1}^{2}} \\
\frac{\alpha}{\alpha_{0}} \bar{\lambda} Y_{1} \frac{\partial \bar{w}_{4}}{\partial X}+\frac{\alpha}{\alpha_{0}} \bar{u}_{1} \frac{\partial \bar{w}_{1}}{\partial X}+\left(\bar{v}_{3}-f_{\bar{z}} \bar{w}_{3}\right) \frac{\partial \bar{w}_{1}}{\partial Y_{1}}+\bar{w}_{1} \frac{\partial \bar{w}_{1}}{\partial \bar{z}}=f_{\bar{z}} \frac{\partial \bar{p}_{6}}{\partial Y_{1}}+\Delta \frac{\partial^{2} \bar{w}_{4}}{\partial Y_{1}^{2}} .
\end{gathered}
$$

In the above, terms that are zero have been omitted. The required contributions from the continuity equation are

$$
\begin{aligned}
& \frac{\alpha}{\alpha_{0}} \frac{\partial u_{1}}{\partial X}+\frac{\partial}{\partial Y_{1}}\left(\bar{v}_{3}-f_{\bar{z}} \bar{w}_{3}\right)+\frac{\partial \bar{w}_{1}}{\partial \bar{z}}=0 \\
& \frac{\partial}{\partial Y_{1}}\left(\bar{v}_{4}-f_{\bar{z}} \bar{w}_{4}\right)+\frac{\partial \bar{w}_{2}}{\partial \bar{z}}+\bar{c}^{\prime}-f_{\bar{x}} \bar{\lambda}=0 .
\end{aligned}
$$


Equations (A5a), (A7a), (A8a) will yield $\bar{v}_{1}, \bar{w}_{1}$ and $\bar{p}_{3}$, and then $\bar{u}_{1}$ and $\bar{v}_{3}-f_{\bar{z}} \bar{w}_{3}$ may be obtained from (A5c), (A9a). Finally, (A7b), (A8b), (A9b) may then be solved for $\bar{v}_{4}, \bar{w}_{4}, \bar{p}_{6}$. We are not in fact interested in $\bar{p}_{3}$ or $\bar{p}_{6}$, and are concerned only with the $E$-independent terms in $\bar{v}_{4}, \bar{w}_{4}$ as these are the terms that lead to (2.14). We carry out this procedure below.

If we eliminate $\bar{p}_{3}$ between (A7a), (A8a) and use (A5a) and write

$$
\bar{w}_{1}=w_{1 E} E+\text { c.c., } \quad \bar{u}_{1}=u_{1 E}+\text { c.c. }, \quad \bar{p}_{1}=p_{1 E} E+\text { c.c. }
$$

we find that $w_{1 E}$ satisfies

$$
\frac{\partial^{2} w_{1 E}}{\partial Y_{1}^{2}}-i a(\bar{x}, \bar{z}) Y_{1} w_{1 E}=\frac{1}{\Delta^{2}} \frac{\partial p_{1 E}}{\partial \bar{z}}
$$

where $a(\bar{x}, \bar{z})$ is defined after (2.14) and is real. The solution of (A11) that is not exponentially large as $\left|Y_{1}\right| \rightarrow \infty$ is

$$
w_{1 E}=\delta(\bar{x}, \bar{z}) \int_{0}^{\infty} \exp \left[-i a^{1 / 3} Y_{1} t-t^{3} / 3\right] d t,
$$

where

$$
\delta(\bar{x}, \bar{z})=-\frac{1}{a^{2 / 3} \Delta^{2}} \frac{\partial p_{1 E}}{\partial \bar{z}} .
$$

Equations (A5c) and (A9b) show that

$$
\frac{\partial^{3} u_{1 E}}{\partial Y_{1}^{3}}-i a(\bar{x}, \bar{z}) Y_{1} \frac{\partial u_{1 E}}{\partial Y_{1}}=-\frac{\bar{\lambda}}{\Delta} \frac{\partial w_{1 E}}{\partial \bar{z}}+\frac{\bar{\lambda}_{\bar{z}}}{\Delta} \frac{\partial}{\partial Y_{1}}\left(Y_{1} w_{1 E}\right),
$$

so that

$$
u_{1 E}=\frac{i}{\alpha} \frac{\partial w_{1 E}}{\partial \bar{z}}-\frac{i}{4 \alpha}\left(\frac{\bar{\lambda}_{\bar{z}}}{\bar{\lambda}}+\frac{a_{\bar{z}}}{a}\right) \frac{\partial}{\partial Y_{1}}\left(Y_{1} w_{1 E}\right) .
$$

It emerges that it is sufficient to obtain $v_{3 E}-f_{\bar{z}} w_{3 E}$ to within an arbitrary function of $\bar{x}, \bar{z}$ from $(\mathrm{A} 9 \mathrm{a})$ as

$$
v_{3 E}-f_{\bar{z}} w_{3 E}=-\left(\frac{\bar{\lambda}_{\bar{z}}}{\bar{\lambda}}+\frac{a_{\bar{z}}}{a}\right) \frac{Y_{1} w_{1 E}}{4}+W_{3 E}(\bar{x}, \bar{z})
$$

where we have used

$$
\frac{\partial w_{1 E}}{\partial \bar{z}}=\frac{\delta_{\bar{z}}}{\delta} w_{1 E}+\frac{a_{\bar{z}}}{3 a} Y_{1} \frac{\partial w_{1 E}}{\partial Y_{1}}
$$

obtainable from (A12).

We require the $E$-independent terms from (A7b), (A8b), so first eliminate $\bar{p}_{6}$ between them and then use $(\mathrm{A} 9 \mathrm{~b})$ which shows that

$$
\partial^{2}\left(\bar{v}_{4}-f_{\bar{z}} \bar{w}_{4}\right) / \partial Y_{1}^{2}=0 .
$$

The required terms in the resulting equation for $\bar{w}_{4}$ are

$$
\frac{\alpha}{\alpha_{0}} \bar{u}_{1} \frac{\partial \bar{w}_{1}}{\partial X}+\left(\bar{v}_{3}-f_{\bar{z}} \bar{w}_{3}\right) \frac{\partial \bar{w}_{1}}{\partial Y_{1}}+\bar{w}_{1} \frac{\partial \bar{w}_{1}}{\partial \bar{z}}+f_{\bar{z}} f_{\bar{z} \bar{z}} \bar{w}_{1} \bar{w}_{1} / \Delta=\Delta \frac{\partial^{2} \bar{w}_{4}}{\partial Y_{1}^{2}} .
$$


The result we require from (A18) is the value of

$$
\int_{-\infty}^{\infty} \frac{\partial^{2} w_{4 N}}{\partial Y_{1}^{2}} d Y_{1}
$$

where $w_{4 N}$ is the $E$-independent component of $\bar{w}_{4}$. The left-hand side of (A18) is

$$
i \alpha u_{1 E}^{*} w_{1 E}+\left(v_{3 E}-f_{\bar{z}} w_{3 E}\right) \frac{\partial w_{1 E}^{*}}{\partial Y_{1}}+w_{1 E} \frac{\partial w_{1 E}^{*}}{\partial \bar{z}}+\frac{f_{\bar{z}} f_{\bar{z} \bar{z}}}{\Delta} w_{1 E} w_{1 E}^{*}+\text { c.c. }
$$

where an asterisk denotes complex conjugate, and upon substitution from (A14), (A15), (A16) we find that

$$
\int_{-\infty}^{\infty} \frac{\partial^{2} w_{4 N}}{\partial Y_{1}^{2}} d Y_{1}=\frac{2}{\Delta}\left\{\frac{\delta_{\bar{z}}}{\delta}+\left(\frac{\delta_{\bar{z}}}{\delta}\right)^{*}+\frac{1}{2} \frac{\Delta_{\bar{z}}}{\Delta}-\frac{1}{3} \frac{a_{\bar{z}}}{a}\right\} \int_{-\infty}^{\infty} w_{1 E} w_{1 E}^{*} d Y_{1}
$$

Since it is easily found from (A12) that

$$
\int_{-\infty}^{\infty} w_{1 E} w_{1 E}^{*} d Y_{1}=\pi\left(\frac{2}{3}\right)^{2 / 3}\left(-\frac{2}{3}\right) ! a^{-1 / 3} \delta \delta^{*}
$$

we have, upon substitution for $\delta$ in (A21) and rearranging the terms, that the difference in $\partial w_{4 N} / \partial Y_{1}$ as $Y_{1}$ increases from minus to plus infinity is given by $J$ as defined in $(2.14 \mathrm{~b})$. The match with (2.7) then gives this expression as the jump in $\partial \bar{w} / \partial \bar{y}$ across the critical level. The jump in $\partial \bar{v} / \partial \bar{y}$ now follows immediately from (A9b). Also the jump in $\bar{q}$ follows from the behaviour of $\bar{p}_{6}$ as $Y_{1} \rightarrow \pm \infty$. The value of this discontinuity is

$$
[\bar{q}]_{-}^{+}=-2 \pi\left(\frac{2}{3}\right)^{2 / 3}\left(-\frac{2}{3}\right) ! \frac{f_{\bar{z} \bar{z}}}{a^{5 / 3} \Delta^{5}}\left|\frac{\partial \tilde{p}}{\partial \bar{z}}\right|^{2} .
$$

from (A8b), (A18), taking (A12b), (A22) into account.

A final comment is that the match between the terms in $Y_{1}$ in $w_{4 N}$ for large $\left|Y_{1}\right|$ and those in $\bar{w}$ as $\bar{y} \rightarrow f(\bar{x}, \bar{z}) \pm 0$ is what determined the orders of magnitude, namely $R^{-1+(5 \delta+b) / 6}$ of the $E$-dependent perturbations in (2.7). In (A2), $\bar{v}_{4}, \bar{w}_{4}$ are forced quadratically by the leading-order perturbations in the critical layer (whose orders of magnitude are related to those of the perturbations in (2.7)). The successful match of $w_{4 N}$ to $\bar{w}$ confirms the choice of the powers of $R$ in (2.7).

\section{References}

N. D. Blackaby (1991). On viscous, inviscid and centrifugal instability mechanisms in compressible boundary layers, including non-linear vortex/wave interactions and the effects of large Mach number on transition. Ph.D. Thesis, University of London.

D. A. R. Davis (1992). On linear and nonlinear instabilities in boundary layers with cross-flow. Ph.D. Thesis, University of London.

J. Gajjar and F. T. Smith (1983). On hypersonic self-induced separation, hydraulic jumps and boundary layers with algebraic growth. Mathematika, 59, 77. 
P. Hall and F. T. Smith (1988). The nonlinear interaction of Tollmien-Schlichting waves and Taylor-Gortler vortices in curved channel flows. Proc. Roy. Soc. Lond. A, 417, 255.

P. Hall and F. T. Smith (1989). Nonlinear Tollmien-Schlichting wave/vortex interaction in boundary layers. Eur. J. Mech., 8, 151.

P. Hall and F. T. Smith (1990). Theory on instability and transition. Proc. ICASE Workshop on Instability and Transition, Vol. II (ed. M. Y. Hussaini and R. G. Voigt) pp. 5-39. Springer.

P. Hall and F. T. Smith (1991). On strongly nonlinear vortex/wave interactions in boundary-layer transition. J. Fluid Mech., 227, 641.

P. S. Klebanoff and K. D. Tidstrom (1959). Evolution of amplified waves leading to transition in a boundary layer with zero pressure gradient. N.A.S.A. Tech. note, no. D-195.

F. T. Smith and P. Blennerhassett (1992). Nonlinear interaction of oblique three-dimensional Tollmien-Schlichting waves and longitudinal vortices in channel flows and boundary layers. Proc. Roy. Soc. Lond. A, 436, 585.

F. T. Smith and R. J. Bodonyi (1985). On short-scale inviscid instabilities in flow past surfacemounted obstacles and other non-parallel motions. Aero. Inl., June/July.

F. T. Smith, S. N. Brown and P. G. Brown (1992). Initiation of three-dimensional transition paths from an inflectional profile. Eur. J. Mech. To appear.

F. T. Smith and A. G. Walton (1989). Nonlinear interaction of near-planar TS waves and longitudinal vortices in boundary-layer transition. Mathematika, 36, 262.

P. A. Stewart and F. T. Smith (1992). Development of three-dimensional nonlinear blow-up from a nearly planar initial disturbance in boundary-layer transition: theory and experimental comparisons. J. Fluid Mech., 244, 79.

A. G. Walton (1991). Theory and computation of three-dimensional nonlinear effects in pipe-flow transition. Ph.D. 'Thesis, University of London.

A. G. Walton and F. T. Smith (1992). Properties of strongly nonlinear vortex/Tollmien-Schlichtingwave interactions. J. Fluid Mech., 244, 649.

Dr. P. G. Brown,

9 Esparto Street,

London. SW18 4DQ

Professor S. N. Brown,

Department of Mathematics, University College London,

Gower Street,

London. WC1E 6BT

Professor F. T. Smith,

Department of Mathematics, University College London,

Gower Street,

London. WC1E 6BT

Dr. S. N. Timoshin,

Department of Mathematics, University College London,

Gower Street,

London. WC 1E 6BT
76D10: FLUID MECHANICS; Incompressible viscous fluids; Boundary-layer theory.

Received on the 26th of May, 1992. 